\title{
Mejorando la reutilización de modelos de simulación de Dinámica de Sistemas
}

Ricardo Sotaquirá Gutiérrez*

Gerly Carolina Ariza Zabala**

* Ph.D. Profesor - Investigador - Director del Grupo en Pensamiento Sistémico - Universidad Autónoma de Bucaramanga - Bucaramanga, Colombia. e-mail:rsotaqui@unab.edu.co

**Ingeniera de Sistemas - Investigadora Grupo en Pensamiento Sistémico - Universidad Autónoma de Bucaramanga - Bucaramanga, Colombia.

e-mail:gariza@unab.edu.co

Fecha de recepción: Marzo 15 de 2005

Fecha de aprobación: Junio 10 de 2005

\section{RESUMEN}

El proceso de difusión de la Dinámica de Sistemas, como metodología para el examen de problemas organizacionales mediante el modelado y la simulación, ha sido comparativamente más lento que el experimentado por otras técnicas gerenciales. Esto puede ser un indicio de que no se ha capitalizado decididamente su caudal de conocimientos prácticos. Estas limitaciones podrían superarse mediante esfuerzos de síntesis y de generalización del vasto cúmulo de casos reales de uso de la Dinámica de Sistemas reportados en la literatura, es decir, a través de la reutilización de modelos en Dinámica de Sistemas. 
En este artículo se establecen, en primer lugar, los logros que hasta ahora se han obtenido en la reutilización de modelos en Dinámica de Sistemas. A partir de este análisis, se demarca un área aún no desarrollada que promete innovaciones metodológicas en la reutilización de modelos de modo que se podrían abrir nuevas puertas de acceso a la Dinámica de Sistemas.

Palabras Clave: Dinámica de sistemas, orientación a objetos, reutilización de modelos, difusión de la dinámica de sistemas.

\section{ABSTRACT}

System Dynamics, the modeling and simulation approach to the study of organizational problems, has experienced a slower diffusion process than has been experienced by other management techniques. This could be a sign of a limited exploitation of its stock of practical knowledge. This constraint could be overcome by synthesizing and generalizing the stock of many real-world applications of the System Dynamics, that is, by reusing System Dynamics models.

This paper identifies current achievements in model reuse in System Dynamics, and from this analysis, a not yet developed area in model reuse is outlined so new access opportunities to System Dynamics could be opened.

Key words: System dynamics, object oriented paradigm, model reusability, diffusion of system dynamics.

\section{INTRODUCCIÓN}

Desde sus orígenes en las décadas de 1950 y 1960 hasta la actualidad, el enfoque de modelado y simulación conocido como Dinámica de Sistemas ha crecido en cantidad de usuarios, en campos de aplicación, en volumen de casos y, en general, se ha difundido a nivel mundial. Una muestra tangible de este crecimiento lo constituyen las conferencias mundiales, que vienen realizándose desde la década de 1980, en donde se presentan anualmente varios cientos de experiencias internacionales. Los primeros trabajos liderados por el Prof. Jay Forrester (1961) estaban orientados a solucionar problemáticas organizacionales $y$, a pesar de que hoy en día se cuenta con un espectro muy amplio de otros campos de aplicación, todavía las organizaciones humanas siguen siendo los objetos estudiados por el mayor número de trabajos en Dinámica de Sistemas.

A pesar de que el número de adeptos a la Dinámica de Sistemas ha seguido creciendo, como metodología para el examen de problemas organizacionales, este proceso de difusión ha sido comparativamente más lento que el experimentado por otras técnicas gerenciales. Por ejemplo, en menos de una década la metodología de medición de desempeño conocida como Balanced Scorecard (BSC) o Cuadro de Mando Integral se ha convertido en una herramienta de uso común, incluso en empresas e instituciones de países en vías de desarrollo, alcanzando así un grado de difusión tal que es inevitable apreciar una cierta lentitud en la divulgación de la Dinámica de Sistemas. En comparación con el BSC, la Dinámica de Sistemas ofrece una metodología con fundamentación matemática que permite un modelado más formal del conocimiento estratégico, un análisis más riguroso de la robustez de las estrategias y la posibilidad de realizar estudios de prospectiva estratégica (Akkermans y Van Oorschot 2002; Ariza y Sotaquirá 2004).

Sería razonable esperar que como resultado del cúmulo de experiencias en el campo organizacional, documentadas en artículos, ponencias, capítulos y libros, se fuese haciendo cada vez más expedito el proceso de modelado organizacional con Dinámica de Sistemas. Sin embargo, su grado limitado de difusión indica que no se ha capitalizado decididamente su caudal de conocimientos prácticos. Mo obstante, en esta historia se puede apreciar una notable 
excepción: los arquetipos sistémicos (Paich 1985; Senge 1990; Wolstenholme 2003).

Precisamente, los arquetipos o estructuras genéricas resultan de un proceso de síntesis de la experiencia acumulada de modelado con Dinámica de Sistemas, especialmente en casos de organizaciones humanas o sistemas sociales.

Fruto de esta síntesis se han venido obteniendo mapas causales, con cierto nivel de abstracción, que explican algunos patrones de comportamiento recurrentes. Este es el conocimiento genérico que capturan los arquetipos. A través de ellos es posible introducir tanto a usuarios experimentados como a novatos en el estudio dinámico-sistémico de una situación organizacional o social. Con esto se ha abierto una nueva puerta de acceso a la Dinámica de Sistemas para muchas personas que no estaban familiarizadas con esta alternativa sistémica. Este fenómeno de difusión se corresponde, al menos parcialmente, con la amplia divulgación de las ideas del libro "La Quinta disciplina" de Peter Senge (1990). Esto demuestra que se pueden superar algunas barreras de acceso a la Dinámica de Sistemas, particularmente en el campo organizacional, mediante esfuerzos de síntesis y de generalización del vasto cúmulo de casos reales de uso de la Dinámica de Sistemas reportados en la literatura.

De manera que, se podría preguntar ¿Además de los arquetipos o estructuras genéricas, qué otros mecanismos de generalización podrían sugerirse para incrementar la difusión de la Dinámica de Sistemas? Desde nuestro punto de vista, los arquetipos son un resultado posible, entre otros, de una práctica más amplia de abstracción, generalización y aprovechamiento del conocimiento acumulado: la reutilización de modelos en Dinámica de Sistemas.

A través de este artículo se explorarán los logros que hasta ahora se han obtenido en la reutilización de modelos en Dinámica de Sistemas. Para ello será necesario construir un marco de referencia que nos permita ubicar las diferentes propuestas que la comunidad dinámico-sistémica ha hecho, explícita o implícitamente, en cuanto a la reusabilidad de modelos. La fuente natural para la construcción de este marco tiene que ser el campo de la Ingeniería de Software, particularmente el paradigma de la orientación a objetos, dado que es allí en donde ha venido creciendo fértilmente el concepto de reutilización. Es decir, los avances en la reutilización del software servirán como referente para situar los avances en la reutilización de modelos en Dinámica de Sistemas (Sotaquirá y Ariza 2004). A partir de este examen metódico del estado del arte, se demarcará y se explorará un área aún no desarrollada que promete innovaciones metodológicas en la reutilización de modelos de modo que se podrían abrir nuevas puertas de acceso a la Dinámica de Sistemas.

\section{REUTILIZACIÓN EN INGENIERÍA DE SOFTWARE ORIENTADA A OBJETOS}

El concepto de reutilización ha venido evolucionando en el campo de la Ingeniería de Software, especialmente a través del paradigma de la Orientación a Objetos. Con el fin de ofrecer una visión organizada de este progreso se propone la siguiente estructura compuesta por tres niveles de reusabilidad.

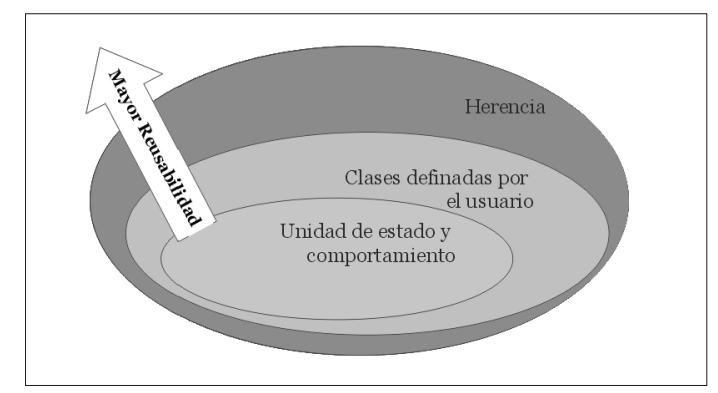

Figura 1. Miveles de reusabilidad en Ingeniería de Software

Cada nuevo nivel implica un mayor potencial de reutilización que el anterior. Las actuales investigaciones en desarrollo de software están 
incrementando estos niveles básicos, pero estos tres niveles constituyen los mínimos compartidos por estándares actuales en este campo.

\subsection{Primer nivel de reusabilidad: unidades débilmente acopladas en estado y comportamiento:}

Usualmente el concepto de objeto, o definido formalmente el concepto de clase, en orientación a objetos es introducido como una solución para la brecha entre datos y código, o entre estado y comportamiento. Esta solución aparece en el contexto de uno de los primeros lenguajes de simulación por computador: Simula (Nygaard 1962; Dahl, Myhrhaug and Mygaard 1968). La primera implementación de Simula, en 1962, contenía una innovación en programación de computadoras. El programa fue organizado en partes independientes que combinaban datos y código: "clientes y servidores". (Simula fue un lenguaje de programación orientado a modelos de colas). Pero en la segunda versión de Simula, se "reemplazaron estas partes con un concepto que puede describir todos los diferentes "objetos" que pueden participar en una simulación. Este concepto fue llamado un "proceso" y puede ocuparse de ambos roles en la simulación, el activo(servidor) y el pasivo(cliente)" (Krogdahl 2003, p.2).

La versión llamada Simula 67 cambió el concepto de procesos por el concepto de clase. El concepto de clase, la definición formal de un objeto, fue la piedra angular para la formulación de un software metodológicamente basado en componentes reusables, ésta fue la primera característica de reusabilidad de software.

Por definición, la Dinámica de Sistemas trasciende la brecha entre estado y comportamiento. Además el modelado en Dinámica de Sistemas no está orientado a construir modelos usando unidades independientes. En efecto los ciclos de realimentación pueden atravesar varios subsistemas o submodelos. Por consiguiente una diferencia concebible entre el concepto de clase en orientación a objetos y el correspondiente concepto en Dinámica de Sistemas es que este último algunas veces corresponde a unidades independientes, pero en el caso general las clases en Dinámica de Sistemas corresponden a unidades escasamente acopladas. Es decir, que ellas pueden ser unidades con un mínimo de acoplamiento entre unas y otras, precisamente con un mínimo de interdependencia para soportar los ciclos de realimentación.

\subsection{Segundo nivel de reusabilidad: clases definidas por el usuario:}

La versión original de Simula ofreció al usuario dos clases básicas predefinidas, servidor y cliente, pero fue solamente hasta Simula 67 cuando aparecieron clases definidas por el usuario. Este evento abrió una basta gama de posibilidades para la reutilización de software. El usuario entonces podía crear nuevas clases compuestas por clases simples definidas previamente por él o por el lenguaje de programación. Por otra parte, un desarrollador puede fácilmente observar la descomposición de un sistema en sus componentes constitutivos, evitando grandes cambios en el sistema completo. El nombre formal para estas dos propiedades del enfoque orientado a objetos son composición y descomposición, respectivamente(Meyer 1988, p.12-13).

Estos atributos del enfoque orientado a objetos son el complemento para el concepto de encapsulación. Un componente puede tener una frontera claramente definida que lo separa en estado y comportamiento de otros componentes, esto es un evidente requerimiento para descomposición. Así los componentes se asemejan a cápsulas. En orientación a objetos (OO) la encapsulación se implementó aplicando el principio ocultamiento de información, por ejemplo en Smalltalk, otro lenguaje orientado a objetos de los años 60, "todo lo que podemos describir puede ser representado por la composición recursiva de un único bloque que oculte su combinación del estado y del proceso 
dentro de sí mismo y que puede ser manipulado sólo a través del intercambio de mensajes"(Kay 1993).

El ocultamiento de información y la encapsulación usualmente implican que los componentes sean cajas negras. Pero recientemente se ha propuesto en $\mathrm{OO}$ un enfoque de cajas transparentes que también satisface los requerimientos de encapsulación y comunicación controlada entre componentes. El enfoque de cajas transparentes puede ser una alternativa apropiada para la encapsulación de clases en SD y esto también ajusta las condiciones de composición y descomposición.

\subsection{Tercer nivel de reusabilidad: jerarquía de herencia y polimorfismo:}

Parecería que un nivel aceptable de reusabilidad es alcanzado cuando la tecnología de software satisface los requerimientos establecidos por los primeros dos niveles de reusabilidad. Pero las "clases, como se han visto hasta aquí, no son suficientes. Ellas proporcionan una buena técnica de descomposición modular.[...]. pero además es necesario alcanzar los objetivos de reusabilidad y extendibilidad. [...]. el progreso en reusabilidad y extendibilidad demanda que se aprovechen las fuertes relaciones conceptuales que se tienen entre clases: una clase puede ser una extensión, una especialización o una combinación de otras. [...]. la herencia proporciona este soporte" (Meyer 1988, p.217).

Obsérvese que las relaciones de herencia y las relaciones de composición son diferentes. En la última, una de las clases es integrada dentro de una nueva clase más compleja. Este tipo de relación entre superclases y subclases equivale a la relación entre el conjunto y las partes que lo componen. Por otro lado, si una subclase hereda de una superclase, esto significa que la subclase será extendida o especializa formas características de esta superclase. La herencia es un tipo de relación padre-hijo. Cuando un lenguaje de programación soporta herencia entonces cualquier actualización en un ancestro es reflejada automáticamente en todos sus descendientes.

El complemento para la implementación de la herencia es la propiedad de polimorfismo. Ésta se refiere a la capacidad de reemplazar una instancia de una clase padre por una instancia de cualquiera de sus clases hijas. Por ejemplo, un sistema organizacional orientado a objetos tiene definida una clase de operación y algunas clases descendientes pueden ser: ventas, marketing y manufactura. Cualquier referencia en el sistema para una instancia de operación puede ser debidamente satisfecha por la instancia de ventas o cualquier otro descendiente.

La jerarquía de herencia y el polimorfismo introdujeron una nueva dimensión de la reusabilidad, éstas constituyen las características más distintivas de la reusabilidad en 00 . Las clases o componentes deberían ser lo suficientemente genéricas para ofrecer un amplio dominio de uso, pero también ellas deberían ofrecer un camino simple para adaptarlas a un uso particular. Este dilema es resuelto mediante la herencia y el polimorfismo.

La aplicación de este nivel de reusabilidad en Dinámica de Sistemas genera la posibilidad de tener estructuras genéricas, pero al mismo tiempo, permite tener una técnica eficiente para ajustar estas estructuras a las circunstancias de un modelo en particular de una forma mucho más robusta y flexible que el enfoque de parametrización anteriormente sugerido por Winch (2002).

\section{ENFOQUES ACTUALES DE REUTILIZACIÓN EN DINÁMICA DE SISTEMAS}

Después de la construcción del marco conceptual de los tres niveles de reusabilidad, es posible ahora volver a la literatura de Dinámica de Sistemas para situar las diferentes propuestas que han aparecido en torno a la aplicación, tácita o 
explícita, del concepto de reusabilidad en Dinámica de Sistemas. Este análisis del estado del arte se sintetiza en la siguiente tabla.

Tabla 1. Estado del arte de la reutilización en Dinámica de Sistemas

\begin{tabular}{|c|c|c|c|}
\hline Arquetipos Sistémicos & Moléculas & Liehr & Powersim y Myrtveit \\
\hline $\begin{array}{l}\text { Los arquetipos sistémicos } \\
\text { son estructuras causales } \\
\text { abstractas, las cuales } \\
\text { presentan poco acopla- } \\
\text { miento de las unidades en } \\
\text { relación con estado y } \\
\text { comportamiento. } \\
\text { A su vez en los arquetipos, } \\
\text { una vez son integrados en } \\
\text { un modelo, pierden sus } \\
\text { fronteras haciendo nula la } \\
\text { composición y descompo- } \\
\text { sición del enfoque de } \\
\text { orientación a objetos. }\end{array}$ & $\begin{array}{l}\text { Las moléculas son estruc- } \\
\text { turas genéricas compues- } \\
\text { tas de elementos de } \\
\text { Dinámica de Sistemas. } \\
\text { Al integrar estas subes- } \\
\text { tructuras en un modelo } \\
\text { pierden sus fronteras } \\
\text { impidiendo así reconocer- } \\
\text { las fácilmente. Además de } \\
\text { lo anterior las moléculas } \\
\text { pretenden integrar la } \\
\text { herencia mediante un } \\
\text { mapa que les da una } \\
\text { organización estructural } \\
\text { pero al momento de } \\
\text { implementarlas éstas no } \\
\text { soportan la jerarquía de } \\
\text { herencia del enfoque de } \\
\text { orientación a objetos. }\end{array}$ & \begin{tabular}{|l|} 
Los componentes de \\
modelos predefinidos \\
están basados en el \\
concepto de estructura \\
genérica. \\
Dichos componentes \\
pueden ser distinguidos al \\
introducirlos en un modelo \\
y pueden ser vistos de \\
manera transparente \\
dependiendo del nivel de \\
agregación en que se \\
encuentre. Los componen- \\
tes de modelos predefini- \\
dos poseen jerarquía es \\
decir, los modelo se \\
componen de submodelos, \\
pero en ningún caso esta \\
jerarquía soporta relaciones \\
padre-hijo.
\end{tabular} & \begin{tabular}{|l|} 
Un componente es una \\
pieza de un modelo que \\
puede ser usada como \\
bloque de construcción \\
para otro componente. \\
Al introducir en un modelo \\
un componente, éste \\
puede ser distinguido del \\
resto del modelo depen- \\
diendo del nivel de jerarquía \\
(top- down) en que se \\
encuentre. \\
En este enfoque no se \\
emplea el concepto de \\
jerarquía de herencia de la \\
orientación a objetos sino, \\
el de jerarquía de compo- \\
sición es decir, los compo- \\
nentes están basados en \\
estructuras básicas, sub- \\
sistemas o sistemas \\
dependiendo del nivel de \\
abstracción que requiera \\
el modelo.
\end{tabular} \\
\hline
\end{tabular}

componentes pueden ser editados y reformulados con base en las necesidades del modelador, o nuevos componentes pueden ser creados a partir de componentes básicos.
Algunos de los enfoques analizados incorporan solamente el primer nivel de reusabilidad, los arquetipos sistémicos (Paich 1985; Senge 1990; Wolstenholme 2003) y las moléculas (Eberlein et. al.1996) pertenecen a este estado. En ambas propuestas, cuando una de estas estructuras es introducida en un modelo pierde la posibilidad de ser distinguida del resto del mismo.

Liehr (2002), Myrtveit (2000) y Powersim (2003) han propuesto otros enfoques con un alto nivel de reusabilidad. En la propuesta teórica de Liehr los componentes predefinidos serían estructuras genéricas que corresponden con entidades del modelo mental.

En el caso de Myrtveit y Powersim la decisión de establecer la correspondencia entre entidades mentales con componentes depende del modelador. En ambos enfoques, cuando este tipo de estructuras son introducidas en un modelo, estas pueden ser diferenciadas de otras estructuras del modelo (enfoque de caja transparente), dependiendo del nivel de agregación. Por otra parte en Powersim estos
Como se mostró en la tabla 1 , tanto Liehr como Myrtviet / Powersim comparten el concepto de jerarquía de composición, una clase de jerarquía diferente a la de herencia. Explícitamente la plataforma propuesta por Liehr se aparta del concepto de herencia o como él lo llama "el principio de herencia" (2002 p.3). Minguna de las dos propuestas satisface los requerimientos impuestos por el tercer nivel de reusabilidad: jerarquía de herencia y polimorfismo.

De modo que gráficamente se puede observar a continuación la ubicación de las propuestas actuales en el marco de referencia de reusabilidad compuesto por tres niveles.

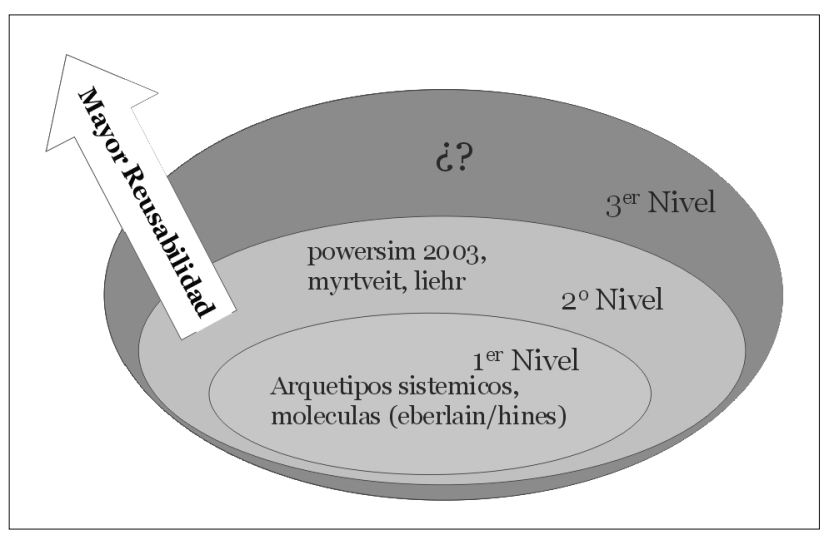

Figura 2. Miveles de reusabilidad en Dinámica de Sistemas

\section{UN NUEVO ENFOQUE DE REUTILIZACIÓN EN DINÁMICA DE SISTEMAS}

En síntesis, se observa que si bien la preocupación por la reusabilidad no constituye una novedad en el modelado con Dinámica de 
Sistemas, sí sería innovador la puesta en práctica, de manera más formal y plena, de todos los niveles de reutilización indicados. Igualmente se resalta que el concepto de jerarquía de herencia es uno de los que ofrece un mayor potencial para fomentar la reutilización de modelos. Con el fin de apreciar mejor este potencial conviene ofrecer una ilustración de las posibilidades que ofrecería la noción de herencia cuando se modela en Dinámica de Sistemas.

Para realizar este sencillo ejemplo se tomará como referencia uno de los modelos organizacionales más clásicos en la literatura de Dinámica de Sistemas. Se trata del conocido modelo del sistema de producción y distribución que aparece en Industrial Dynamics de Forrester (1961). Este sistema contempla el flujo de pedidos y productos entre tres instancias del sistema: la fábrica, los distribuidores y los minoristas (op.cit., p. 137).

Antes de presentar un fragmento de este modelo, cabe destacar que Forrester reconoce que hay semejanzas evidentes entre los submodelos que lo componen. Literalmente expresa que: "En este análisis los tres sectores minorista, distribuidor y fábrica serán muy similares entre sí" (Ibíd., p.139). Más adelante indica que el modelo matemático del minorista es lo suficientemente general para servir de base al resto del modelo. Incluso en esa primera versión que aparece en el ejemplo, Forrester opta por establecer para el distribuidor un conjunto de ecuaciones "idénticas en forma" (Ibíd., p.158) a las del minorista. Es claro que estas semejanzas estructurales precisamente sugieren la aplicación de un enfoque de modelado que se beneficie de ellas a través de algún mecanismo conceptual orientado a la reutilización. Éste fue uno de los motivos que orientaron la selección de este ejemplo para ilustrar el modelado en Dinámica de Sistemas enriquecido con conceptos de la orientación a objetos.

El submodelo que representa al minorista está compuesto por tres niveles y dieciocho ecuaciones. Forrester inicia el proceso de modelado mostrando las relaciones básicas entre los dos niveles principales: el de inventario de productos y el de órdenes pendientes. Para ilustrar el potencial de la reusabilidad basta con circunscribirse tan sólo a ese fragmento del modelo. Su diagrama de niveles y flujos se muestra en la siguiente figura.

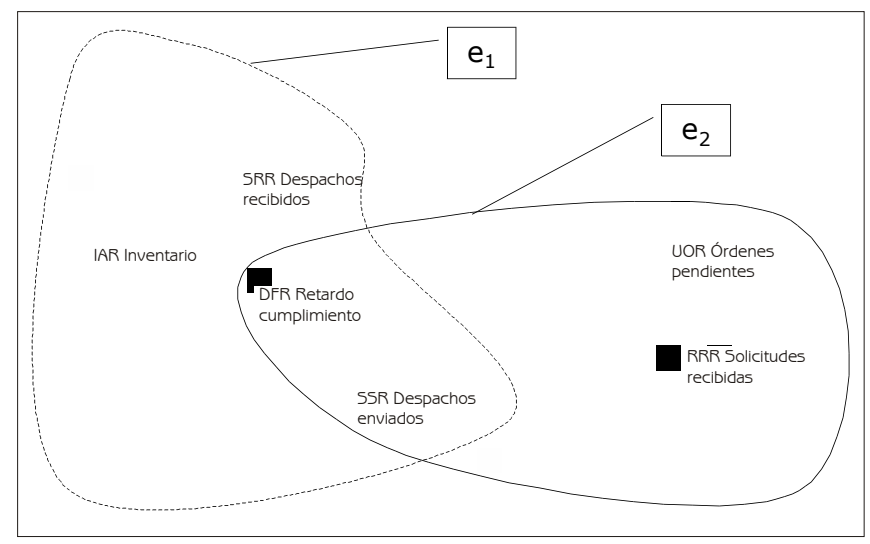

Figura 3. Fragmento del modelo del minorista

El inventario de productos se incrementa con el arribo de Despachos recibidos y disminuye debido a los Despachos enviados. Algo similar ocurre con las Órdenes pendientes y sus correspondientes flujos. Mótese que ambos comparten el valor de la tasa de salida denominada Despachos enviados. Para que el fragmento no quede indefinido se está asumiendo inicialmente en este ejemplo que el Retardo cumplimiento, es decir, la demora para satisfacer los pendientes, es una constante. Ésta podría ser plausiblemente una situación común cuando se está modelando en Dinámica de Sistemas y se comienza con simplificaciones mayores de la complejidad para luego, gradualmente, dejar atrás tales restricciones y agregar complejidad a la representación.

Si este ejercicio de modelado se viera influenciado por las nociones de reusabilidad antes expuestas sería razonable notar en el

Volumen 7 - No. 1 - Julio de 2005 
diagrama que este modelo está constituido por dos estructuras, $e_{1}$ y $e_{2}$, muy semejantes, como se señala en la figura. Ambas estructuras están constituidas por un nivel con sus respectivos flujos de entrada y de salida, y más aún, el flujo de salida está definido de manera idéntica en ambos casos. En términos de la orientación a objetos el modelo se reformularía. En primer lugar debería construirse una clase o un componente definido por la estructura común que se acaba de identificar y que se denominará e. Luego simplemente se establecería que tanto $e_{1}$ como $e_{2}$ son descendientes de e (heredan su estructura y comportamiento).

Pero, a la vez, cuando se elabore el respectivo submodelo del distribuidor, siguiendo a Forrester, se obtendrá un diagrama idéntico al de la figura 3. De modo que ese submodelo, compuesto por las estructuras $e_{1} y_{2}$, puede verse a la vez como una estructura $E$ que es común al minorista y al distribuidor. Es decir, el correspondiente submodelo de cada uno de ellos sería simplemente un descendiente de $E$. El lector puede revisar el citado modelo de Forrester y apreciar, siguiendo su propia iniciativa, más ilustraciones de las posibilidades de reutilización que allí se presentan.

Pero el potencial del concepto de jerarquía de herencia se despliega con mayor claridad a continuación. Supóngase que, en una posterior iteración del proceso de modelado, se decide que la demora en el cumplimiento no será más una constante sino, para representar mejor la situación industrial en cuestión, y que será ahora inversamente proporcional al inventario disponible, como se indica en la fiqura.

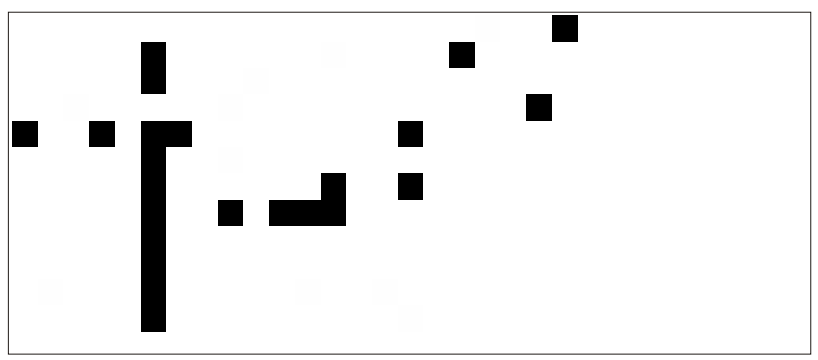

Figura 4. Mejoras a la representación del retardo en el cumplimiento
Si se estuviese modelando bajo un enfoque que soporte esta reutilización, es decir, que tenga la capacidad de operacionalizar la noción de herencia, entonces sería suficiente con modificar el elemento Retardo cumplimiento y las relaciones correspondientes en la estructura que se denominó e. Gracias a que $e_{1}$ y $e_{2}$ son descendientes de ella, automáticamente estas estructuras quedarían actualizadas. Y dado que ambas componen a $E$, y que los submodelos minorista y distribuidor se derivan de esta última, entonces la mejora se propagaría implícitamente a todo el modelo de ejemplo. En cambio de repetir este cambio en seis ocasiones, bastaría con hacerlo una única vez, con lo cual se evitarían adicionalmente posibles errores. Ésta es una ilustración de los beneficios que traería la aplicación del concepto de herencia y, en general, del enfoque de la orientación a objetos en términos de la reutilización de modelos en Dinámica de Sistemas.

\section{CONCLUSIONES}

Para enfrentar la problemática de la escasa difusión de la Dinámica de Sistemas se ha tomado como referencia el enfoque de la orientación a objetos y particularmente su noción de reutilización. Posteriormente se demostró, a través de una revisión del estado del arte de la reusabilidad en Dinámica de Sistemas, que ninguna de las propuestas actuales cumple con los requerimientos de la reutilización orientada a objetos. Especialmente se observó que los trabajos previos no aplican el concepto de jerarquía de herencia. Luego, a través de un ejemplo, se ha intentado ilustrar en qué consistiría un modelado con Dinámica de Sistemas que hiciera uso del concepto de herencia.

Las bondades de este nuevo enfoque podrían ponderarse desde dos perspectivas ciertamente distintas. Desde el punto de vista del practicante actual de la Dinámica de Sistemas los beneficios del enfoque son similares a los percibidos por un Ingeniero de Software que aplica la OO: se logra 
un nuevo nivel de modularidad en el modelado que facilita su organización y la actualización iterativa de los modelos, pero que especialmente hace posible acelerar el proceso de construcción una vez se vayan teniendo componentes genéricos que son reutilizados y fácilmente actualizables. Estos beneficios se ampliarían si se piensa en que toda una comunidad de modeladores utilice este enfoque.

Por otro lado, desde el punto de vista de los posibles usuarios en empresas y organizaciones, la posibilidad de contar con componentes previamente construidos y probados podría facilitar y agilizar la construcción de modelos propios y con ello promover el uso de la Dinámica de Sistemas en el ámbito organizacional.

Mo obstante, también debe reconocerse una restricción importante de este enfoque de modelado soportado en la reutilización. Se sabe que en Dinámica de Sistemas se genera un proceso de aprendizaje en quienes participan en la construcción y uso de los modelos (Sotaquirá et. al. 2000). Pero aquellos usuarios que no participaron en la construcción de los componentes, han sido privados de una cierta experiencia formativa. Sin embargo, esta limitación puede mitigarse en la medida en que ellos puedan conocer la estructura interna de tales componentes, es decir, que éstos tendrían que ser submodelos de caja transparente (Machuca 2000).

Por último, para catalizar esta mejora metodológica parece necesaria una herramienta software de modelado que soporte este enfoque. En la actualidad nuestro grupo de investigación viene llevando a cabo un proyecto que busca obtener un primer prototipo de tal herramienta.

\section{Agradecimientos}

Los autores agradecen a las entidades que financian esta investigación: Colciencias, Universidad Autónoma de Bucaramanga y Pensemos Ltda. Igualmente expresan su gratitud al equipo de desarrollo de software de Pensemos Ltda. por su vital participación en este proyecto.

\section{REFERENCIAS BIBLIOGRÁFICAS}

1. Akkermans H, Van Oorschot K. 2002. Developing and Balanced Scorecard with System Dynamics, Proceedings of the 20th International System Dynamics Conference(CD-ROM), Palermo, Italy. July. Systems Dynamics Society.

2.Ariza G, Sotaquirá R. 2004. Un Muevo Enfoque de Modelado de Estrategias Empresariales con Dinámica de Sistemas, Memorias del Segundo Encuentro Colombiano de Dinámica de Sistemas, Santa Marta, Colombia. Ediciones UIS.

3.Dahl O, Myhrhaug B, Mygaard K. 1968. SIMULA 67 Common Base Language, Morwegian Computing Center.

4.Eberlein RJ, Hines JH. 1996. Molecules for Modelers, Proceedings of the 14th International System Dynamics Conference (CD-ROM), Cambridge, Massachusetts, USA. July. System Dynamics Society.

5.Forrester J. 1961. Industrial Dynamics. Cambridge Press.

6. Kay A. 1993. The Early History of Smalltalk, ACM SIGPLAM Motices, 28(3): 69-95, ACM.

7.Krogdahl 5. 2003. The Birth of Simula, Proccedings of the HirC 1 Conference, Trondheim, June. IFIP.

8.Liehr M. 2002. A Platform for Systems Dynamics Modeling: Methodologies for the Use of Predefined model Components, Proceedings of the 20th International System Dynamics Conference(CD-ROM), Palermo, Italy. July. Systems Dynamics Society.

9.Machuca, J. 2000. Transparent-box business simulators: An aid to manage the complexity of organizations, Simulation \& Gaming, 31(2): 230-239.

10.Meyer B. 1988. Object-Oriented Software Construction, 1st edn. Prentice Hall International (UK) Ltd.

11. Myrtveit M. 2000. Object Oriented Extensions 
to System Dynamics, Proceedings of the 18 th International System Dynamics Conference(CD_ROM), Bergen, Morway, August. System Dynamics Society.

12. Mygaard K. 1962. SIMULA: An Extension of ALGOL to the Description of Discrete-Event Metworks, Proceedings of the IFIP congress 62, Munich, Morth-Holland Publ.

13.Paich, M. 1985. Generic Structures, System Dynamics Review, 1: 126-132.

14.Powersim. 2003. Powersim Studio Express 2003 Online Help. Powersim AS.

15.Senge PM. 1990. The Fifth Discipline. Doubleday.

16. Sotaquirá R., Gélvez L., Cabrera J. 2000. Hacia un mirar latinoamericano de la aplicación de la Dinámica de Sistemas en estudios organizacionales, Heurística, 10: 48-59.

17. Sotaquirá R, Ariza G. 2004. Reusability in System Dynamics: Current approaches and improvement opportunities, Proceedings of the 22th International System Dynamics Conference(CD_ROM), Oxford, England, July. System Dynamics Society.

18. Winch G. 2002. User-parameterised generic models: a solution to the conundrum of modelling access for SMEs?, System Dynamics Review, 18(3): 339-357.

19. Wolstenholme, E. 2003. Towards the definition and use of a core set of archetypal structures in System Dynamics, System Dynamics Review, 19(1): 7-26. 